\title{
Causes of periodical rainfall distribution and long-term forecast of precipitation for Lankaran, Azerbaijan
}

\author{
Asger Mammadov, Rustam Rajabov, Naila Hasanova \\ Baku State University, Academic Zahid Khalilov Street 23, AZ 1148, Baku, Republic of Azerbaijan, e-mail: asger_mam- \\ madov@mail.ru,rajabov_r_f@mail.ru;naila.hasanova@mail.ru
}

\begin{abstract}
Irregular rainfall distribution is receiving considerable attention. The amount of rainfall for one region can account for 500-600 mm, sometimes $1000 \mathrm{~mm}$. For example, in the year 1985, Zagtala in Azerbaijan received $716 \mathrm{~mm}$ of rainfall, while in 1988, 2004 and $2008-1151,1306$ and $661 \mathrm{~mm}$, respectively, were measured. In Lankaran $2061 \mathrm{~mm}$ were measured in 1982 and $470 \mathrm{~mm}$ in 1999. Generally, rainfall distribution differs across the Republic of Azerbaijan. In this study, the physical side of such variations was clarified. In relation to that, the movement speed of the atmosphere in regard of the rotation of the Earth was analyzed, showing that the difference in rainfall distribution, according to the time structure, is connected to the direction change of the atmospheric movement. Generally, the reasons for atmospheric movements cannot be identified as the rotation movement of the earth, mainly because both environments show different activities. While the processes happening in the atmosphere often change, influenced by the pressure gradient, the rotation movement of the earth is more stationary.

We also evaluated the rainfall forecast method for the region Lankaran. Taking into account its simplicity, the Shuster method was used. Observation data was divided into stationary and casual elements. Selection of periodicals was determined by separation of long term meteorological data into harmonic functions. By accepting the variation, the coefficient casual item was added.
\end{abstract}

Keywords: rainfall distribution, synoptic processes, atmospheric forces, harmonic analysis, baric gradient

Submitted 11 February 2015, revised 23 March 2017, accepted 5 April 2018

\section{Introduction}

The spatial and temporal distribution patterns of precipitation are relatively complex. Some regions receive rain each day, while in others, there is no precipitation for several years. Even within a radius of $100 \mathrm{~km}$, rainfall distribution patterns differ. Thus, the fluctuation of precipitation distribution is larger than the climate norms, and in some regions, the annual precipitation sum differs from the norm by about 500-600 $\mathrm{mm}$.

Rainfall distribution slightly differs from the perennial norm in each area and year. Such differences can range from 200 to $300 \mathrm{~mm}$ in flat areas and from 500 to $600 \mathrm{~mm}$ in mountainous regions. Since the territory of Azerbaijan has complex relief features, the differences in rainfall distribution are not surprising. This type of diversity is common in any region of the Earth.

This article proposes a methodology for perennial prediction of rainfall distributions, using Lankaran as a case study. The overall aim of this study is to identify a new approach that explains the spatial variation in rainfall distribution, presenting a new methodology for rainfall prediction.

\section{Research area}

The natural district of Lankaran is situated in the southeast of Azerbaijan. According to its relief and geological structure, Lankaran can be divided into mountainous and plain regions. Two-third of the territory is mountainous, while one-third is plain. Due to the complex physical-geographical features of the district, it can be characterized as follows: 1. mild hot climate with dry summers (mainly on the Lankaran plain); 2. cold climate with dry winters (mainly in areas above $1400 \mathrm{~m}$ sea level); 3. semi-desert and dry field climate (mainly in areas above $1000 \mathrm{~m}$ ). The climate of the Lankaran plain is humid subtropical, with mild winters, hot and dry summers, and wet autumns. Average annual temperature is $14^{\circ} \mathrm{C}$. The coldest and hottest months are January $\left(+3,4^{\circ} \mathrm{C}\right)$ and July $\left(+25^{\circ} \mathrm{C}\right)$, respectively. Annual rainfall ranges between 1400 and $1600 \mathrm{~mm}$. Average annual temperature of the mountainous regions is $11,9^{\circ} \mathrm{C}$, with annual rainfall of $300-400 \mathrm{~mm}$. In these regions, average temperatures in January and July are -3 to $-5^{\circ} \mathrm{C}$ and 15 to $20^{\circ} \mathrm{C}$, respectively (Fig. 1). 


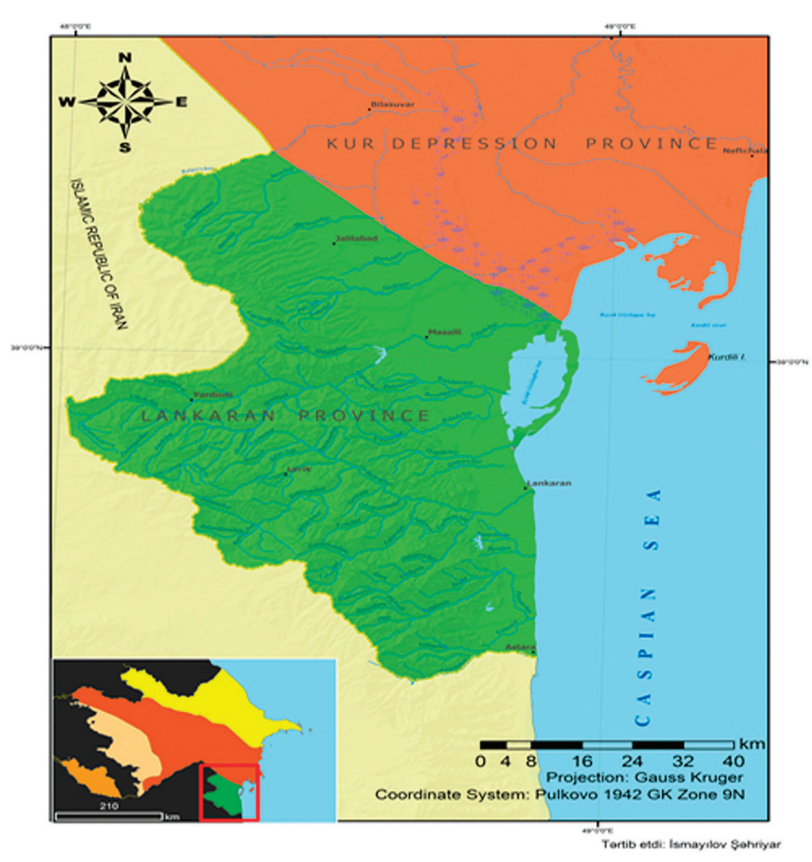

Figure 1. Schematic scheme of the natural district of Lankaran

\section{Methods and data}

Atmospheric changes are a result of the Earth's rotational movement. However, the forces influencing the atmosphere also influence its movement direction in relation to the Earth. Influenced by these forces, the speed of the atmospheric movement, in relation to the Earth, increases and decreases. The baric gradient force is playing great role in the forming of physical processes in atmosphere. Thus, if the direction of the gradient force in the atmosphere coincides with the direction of the atmospheric movement, the atmospheric movement is faster than the rotational movement of the Earth; in the opposite case, the atmospheric movement is slower than the rotational movement. As a result of this movement over the surface of the Earth, the east wind blows at low latitudes, while the west wind blows at moderate and high latitudes. While the movement of the atmosphere at low latitudes is delayed, to some degree, in respect to the rotation of the Earth, at moderate and high latitudes, the atmospheric movement is slightly faster than the rotational movement of the Earth. The impulse momentum of the east wind is negative, but positive for the west wind. With an increasing impulse momentum, i.e., at escalating west winds, the rotational movement of the Earth weakens. When the impulse momentum of the atmosphere weakens, the east wind becomes stronger (and the west wind weakens); consequently, the rotational movement of the Earth increases (Shver 1975). According to the natural law of conservation and movement quantity of forces influencing the atmosphere, the movement of the atmosphere, in respect to the rotational movement of the Earth, can be delayed or hastened (depending on the value and direction of the baric gradient force).

The effects of centrifugal and friction forces are low at high latitudes, such as $1,5 \mathrm{~km}$ above the boundary layer, they are not considered. The value of the centrifugal force on the Earth surface is stable; however, the friction force depends mainly on the speed of the wind (in relation to the Earth's atmosphere) and on orographic features. The Coriolis force also depends on geographical latitudes and the relative movement speed. It is also necessary to take into account that the value of the friction force is greater on dry surfaces than on water surfaces. Therefore, with the complexity of orographic features, the value of the friction force increases at high latitudes, and the atmosphere movement breaks more (ground surface) at high latitudes.

The baric gradient force is the main force in the momentum of air masses. In the formation of atmospheric processes, the role of this force is significant. Under the influence of these forces, the direction of the wind changes frequently, and clouds and rains are transferred to other parts of the Earth. The value of the baric gradient force depends on orographic features and is compensated by friction and Coriolis forces. Unequal precipitation distribution is also connected to the baric gradient force. Value changes of this force are determined by the temperature contrast between the upper and the lower latitudes. While the temperature contrast is high, the impact of advective processes increases in the region; on the contrary, the value of the contrast is small and convective processes are dominant. A frequent change of temperature contrast causes a change in the direction of the baric gradient vector; as a result, the transfer of atmospheric precipitation changes over time. The surface cover around the equatorial regions is more homogeneous than at other latitudes; about $20 \%$ of the land area are dry, while the rest of the surface is water. Here, the value of the friction force is less changeable and relatively weak at higher latitudes, where the Coriolis force is extremely small. The homogeneity of the surface affects the changes in the baric gradient force and has a reducing effect. One of the reasons for high precipitation at this latitude is the high humidity and slight change in the baric gradient force. At the same time, the vector of the baric gradient force, from moderate latitudes towards the equator, can be regarded as a quantitative factor that causes higher precipitation levels at moderate latitudes. In some areas, $80 \%$ of the precipitation is characterized as advection precipitation (Bagirov 1965). Within a narrow zone around the equator, the amount of precipitation throughout the year is $1500-2000 \mathrm{~mm}$. On the east coast of Africa, precipita- 

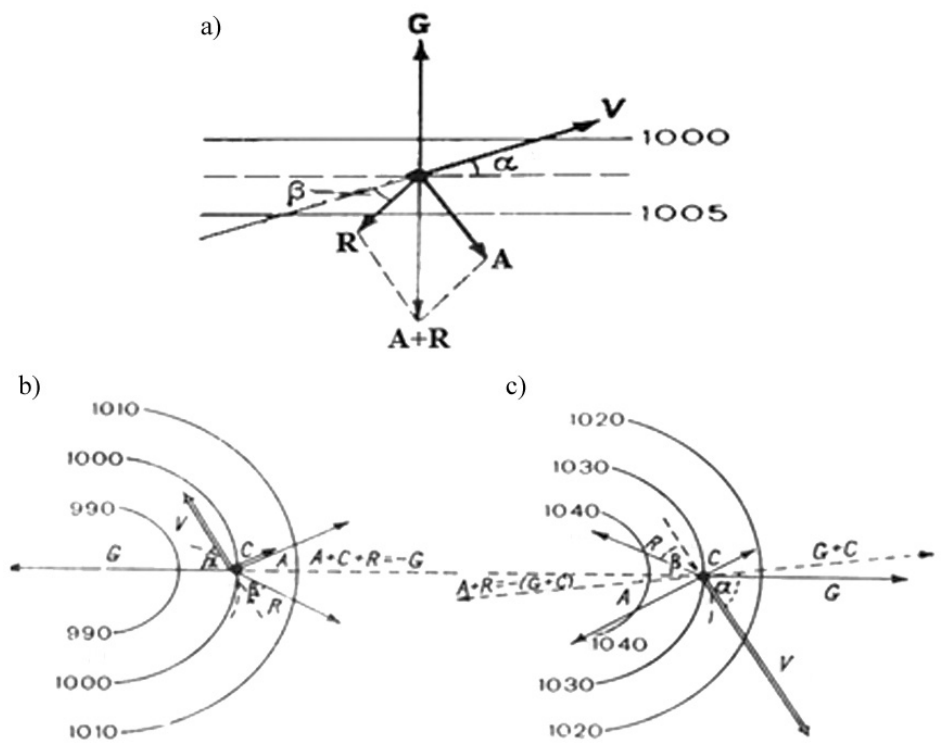

Fig. 2. Mutual state of the forces affecting the atmosphere, taking into account the friction force; a-movement in isobaric rectangular processes, $\mathrm{b}$ - movement in cyclonic processes, and $\mathrm{c}$ - movement in anti-cyclonic processes

tion is less than $1000 \mathrm{~mm}$, while on the islands of Borneo and Sumatra, as well as the upper parts of the Amazon River, precipitation levels account for up to $3000 \mathrm{~mm}$ per year. Differences in precipitation distribution in different regions depend on the orographic characteristics of the area. In general, the differences in the spatial and temporal structure in the regions around the equator are smaller than at other latitudes.

The impacts of friction and Coriolis forces increase towards high latitudes, and the influence of the baric gradient force and the rotational movement of the atmosphere, in respect to the rotation of the Earth, change significantly. The rotational movement of the atmosphere in respect to that of the Earth is slightly delayed. For this reason, precipitation amounts in the western coastal regions are lower than those in the eastern coastal regions. Although the Sahara Desert stretches to the shores of the Atlantic Ocean in the northern hemisphere, precipitation is lower and does not exceed $250 \mathrm{~mm}$ per year. The west coast of North America, especially from the Californian peninsula and further north, is characterized by a low amount of precipitation. In the eastern part of North America, a corresponding arid region does not exist. In general, according to the general laws adopted for trade wind zones, precipitation is lower on the west coast than on the east coast. On the contrary, the situation varies in moderate zones, where the west coast is relatively more humid than the east coast. On the coast of South America, from $32^{\circ}$ latitude to the west, precipitation increases to up to $2000 \mathrm{~mm}$.

However, in this area, precipitation only occurs in a relatively small area, on the western slopes of the Andes Mountains, and decreases drastically to less than $200 \mathrm{~mm}$ per year.
Thus, one of the reasons for the unequal distribution of temporal and spatial precipitation are changes in the value and the direction of the baric gradient force. In addition, it should be noted that in the upper layers, from the boundary layer of the atmospheric movement occurs under geostrophic regularity. This process forming by mutual influence of the baric gradient with Coriolis force.

In contrast to the Earth, the atmosphere is relatively less stable and more homogeneous. Therefore, the direction of the air speed, in a very rare case and for a short period of time, may correspond with the direction of the rotational movement of the Earth. Figure 2 shows the effect directions that cause atmospheric movement during three different natural processes. In all three processes, the centrifugal force is perpendicular to the speed vector of the wind, and the Coriolis force corresponds with the force vector in cyclones, although its direction is opposite in anticyclones.

As one of the reasons for precipitation distribution in continents, atmospheric circulation receives considerable interest [Mamedov et al. 2010]. In a large part of the Eurasian continent, moving away from the Atlantic Ocean from the west coast to the east, the annual amount of precipitation decreases. However, in some areas, violation of this rule only takes place in mountainous areas. In this case, influenced by the baric gradient force, the atmospheric movement breaks, resulting in a delayed speed in respect to the rotation of the Earth. As a result, water evaporated from the surface of the ocean does not reach the dry surface, because its speed is relatively high.

The precipitation amount is not only affected by horizontal transfer, but also depends on vertical processes. In this regard, areas where cyclones frequently occur 
receive high amounts of rainfall, whereas in other regions, rainfall is lower. In addition, under unstable stratification conditions, i.e., due to thermal convection, rainfall events may occur.

Thermal convection is formed while the surface of the Earth heats, but not all shielding movements can form precipitation. In arid areas of Russia (southern and south-eastern regions), the dry surface and the air layer above it heat in summer and the intensity of the raising air masses increases, albeit without cloud formation and rain. This can be explained by the lack of a humidity capacity of the air at high temperatures and by the low relative humidity [Khvichiya et al. 1971]. At the same time, it should be noted that here, it is impossible to ignore the impact of the baric gradient force. Evaporated humid air mass in dry places moves to other places by Baric gradient force.

\section{Proposed estimation method for defining for periodicals}

In this context, the structure of the precipitation distribution is more complex, and precipitation is more difficult to forecast. By taking into consideration the mentioned difficulties, the precipitation forecast for one of the major economic regions of Azerbaijan (Lankaran region) was studied. For this, precipitation data from 1900 Lankaran synoptic observation stations were used.

The row of this data is divided to the harmonic series, and periodicals are chosen. Periodicals are determined by the following analytical expression:

$$
y=A_{1} \sin \left(\frac{2 \pi}{\tau_{1}} t+\varphi_{1}\right)
$$

where:

$$
\begin{gathered}
A_{1}=\sqrt{a^{2}+b^{2}}, \varphi_{1}=\operatorname{arctg} \frac{a}{b} \\
a=\frac{2}{\tau_{c}} \sum_{i=1}^{\tau_{c}} y \cos \frac{2}{\tau_{c}} t, \quad b=\frac{2}{\tau_{c}} \sum_{i=1}^{\tau_{c}} y \sin \frac{2 \pi}{\tau_{c}} t
\end{gathered}
$$

There are different periodical functions in the structures of each row. Therefore, the analytical expression of the row is as follows:

$$
\begin{gathered}
y=A_{0}+A_{1} \sin \left(\frac{2 \pi}{\tau_{1}} t+\varphi_{1}\right)+A_{2} \sin \left(\frac{2 \pi}{\tau_{2}} t+\varphi_{2}\right)+\ldots \\
\ldots+A_{n} \sin \left(\frac{2 \pi}{\tau_{n}} t+\varphi_{n}\right)+\ldots+\theta
\end{gathered}
$$

where $\theta$ is the random section in the given line.
The results of these calculations, based on the described method, are shown in Figure 3.

\section{Results}

The results of analyses are shown in Fig. 3. Thus, for the first time, rainfall delay was forecasted on the base of a stochastic model for the natural district of Lankaran, one of the economic regions of Azerbaijan. Currently, based on this method, droughts are forecasted for different regions of the Republic. Although currently, such forecasts only have a qualitative character, basic elements of their quantitative indices are already determined.

Based on such forecasts, rainfall will increase to 100-150 mm until 2019 and will be $100 \mathrm{~mm}$ above the norm in 2020-2025. Between 2025 and 2040, it will decrease by $100-150 \mathrm{~mm}$ in the mentioned region, and in 2022, 2030 and 2040 it will be $100 \mathrm{~mm}$ above the norm. From 2040 to 2050 rainfall will be about $50 \mathrm{~mm}$ above the norm. Based on this method, we consider that, with changing rainfall patterns, severe after-effects of droughts, compromising agricultural production, will be minimized.

Figure 4 shows the decrease in rainfall during the investigation period. Based on a comparison of Fig. 2 and 3 , we see the conformity of information between calculated and observed data, indicating satisfactory results of the predictions.

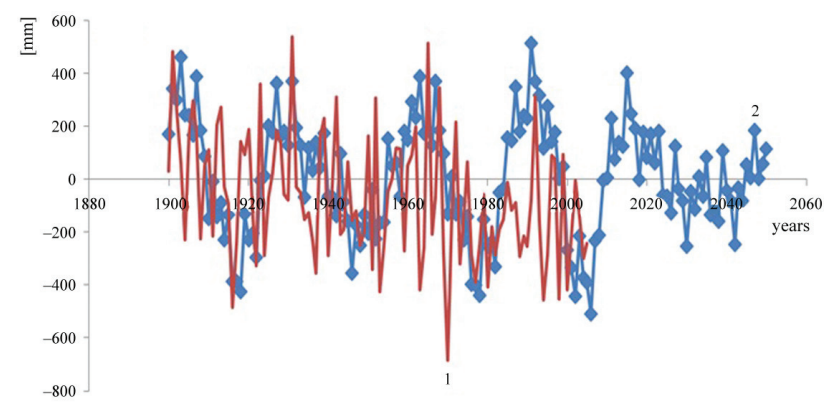

Fig. 3. Actual and estimated coefficients of rainfall fluctuations in Lankaran between 1900 and 2050

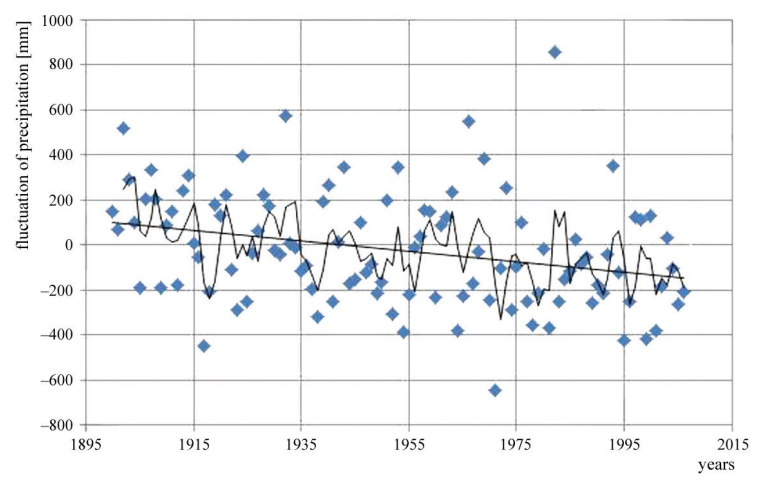

Fig. 4. Perennial dynamics of fluctuation of precipitation in Lankaran 
Bibliography

Bagirov N.A., 1965, On monthly rainfall distributions, (Scientific Works), Sï, 130, 3-21

Mamedov A.S., Mamedova A.R., 2010, Space-time structure of atmospheric rainfall in Azerbaijan territory, «Poisk», Natural and Technical Sciences, 1, 153-158

Khvichiya M.S., 1971, Atmosphere rainfall: climate resources of Georgia, ZakNIQMI, 44 (50), 262-271

Shver T.A., 1975, Unequal rainfall distribution as the main landscape indicator, L.: BGR (Scientific works), 341, 87-97 
